\title{
Buddha as an eye opener: a link between prosocial attitude and attentional control
}

\author{
Lorenza S. Colzato ${ }^{*}$, Bernhard Hommel' ${ }^{1}$, Wery P. M. van den Wildenberg ${ }^{2}$ and Shulan Hsieh ${ }^{3,4}$ \\ Cognitive Psychology Unit and Leiden Institute for Brain and Cognition, Leiden University, Leiden, Netherlands \\ 2 Amsterdam Center for the Study of Adaptive Control in Brain and Behaviour (Acacia) Psychology Department, University of Amsterdam, Amsterdam, Netherlands \\ ${ }^{3}$ Department of Psychology and Institute of Cognitive Science, National Cheng Kung University, Taiwan City, Taiwan \\ ${ }_{4}^{4}$ Department of Psychology and Institute of Allied Health Sciences, National Cheng Kung University, Taiwan City, Taiwan
}

Edited by:

Henk Barendregt, Radboud University, Netherlands

\section{Reviewed by:}

Antonino Raffone, Sapienza University of Rome, Italy

Antoine Lutz, Waisman Lab for Brain

Imaging and Behavior, USA

Paul Van den Hurk, Radboud University,

Netherlands

\section{*Correspondence:}

Lorenza S. Colzato, Department of

Psychology, Cognitive Psychology Unit,

Leiden University, Postbus 9555, 2300

RB Leiden, Netherlands.

e-mail: colzato@fsw.leidenuniv.nl
Increasing evidence suggests that religious practice induces systematic biases in attentional control. We used Navon's global-local task to compare attentional bias in Taiwanese Zen Buddhists and Taiwanese atheists; two groups brought up in the same country and culture and matched with respect to race, intelligence, sex, and age. Given the Buddhist emphasis on compassion for the physical and social environment, we expected a more global bias in Buddhist than in Atheist participants. In line with these expectations, Buddhists showed a larger globalprecedence effect and increased interference from global distracters when processing local information. This pattern reinforces the idea that people's attentional processing style reflects biases rewarded by their religious practices.

Keywords: Buddhism, attention, global precedence

\section{INTRODUCTION}

Human behavior is commonly proactive rather than reactive: We carry out planned actions to reach particular goals rather than wait for environmental events to get us moving. Doing so calls for executive-control functions that configure our cognitive system in such a way that information processing is tailored to the task at hand. Exerting executive control has been compared to parameterizing processing routines in a computer program and a number of possible control parameters have been suggested (Logan and Gordon, 2001). One of these parameters (Logan and Gordon's "c") is assumed to control the grain size of perceptual analysis, that is, whether one is attending more to global or more to local aspects of stimulus events (Logan, 1996).

Investigations of executive-control operations commonly involve instructing participants to prepare for arbitrarily chosen tasks and/or to switch from one task to the other to study the efficiency, characteristics, and time costs of implementing the respective control parameters. The general assumption is that the parameterization operation proper drives the efficiency of cognitive control, whereas the particular parameter values are less important and can be chosen arbitrarily by the system. However, a number of recent studies suggest that this might be an oversimplification, as individuals and groups seem to have relatively stable preferences for particular parameter values. Most of the evidence comes from studies using versions of Navon's (1977) global-local task. In this task, participants are presented with hierarchically constructed stimuli that can be analyzed at two different levels at least, such as larger letters or shapes made of smaller letters or shapes (e.g., a large H made of small S's). Participants are commonly faster and more accurate to respond to the global than to the local level of these stimuli - the so-called global-precedence effect (Navon, 1977). However, the size of this global-precedence effect (i.e., the difference in reaction times and/or error rates when responding to the local vs. the global level) can differ substantially between individuals or groups.

For instance, Masuda and Nisbett (2001) reported significantly smaller global-precedence effects for people raised in the NorthAmerican culture than for people raised in Japan. The authors have attributed this observation to the fact that Western cultures often emphasize the individual and individual goals and needs, whereas East Asian cultures emphasize the importance of the group and the social embedding of the individual in the group context (Nisbett and Miyamoto, 2005). Along the same line, McKone et al. (2010) found that, relative to Caucasians, first and second generations of East Asian immigrants in a Western society showed a strong global advantage. Notably, systematic biases in attentional-control parameters have been demonstrated even between groups that are embedded in the same society. Colzato et al. (2008) reported that Dutch Calvinists show significantly smaller global-precedence effects than Dutch atheists although the two groups were fully matched in terms of race, intelligence, sex, age, and a number of personality variables. As the authors suggested, this difference might reflect the cognitive implications of the most central neo-Calvinist concept: the sphere sovereignty principle that is responsible for the rigorous "pillarization" (segregation) of Dutch and South-African societies for several decades (Boesak, 1984; Bratt, 1998). If so, one would expect a more pronounced global-precedence effect in members of religions that emphasize solidarity and the social responsibility of individuals, such as Catholicism and Judaism. Indeed, this hypothesis was confirmed in recent comparisons that showed a global preference in Roman Catholics (relative to seculars in Italy) and in Orthodox Jews (in comparison to seculars in Israel, Colzato et al., in press). 
The findings above suggest that cultural and religious practice might induce a tendency to prefer one particular control-parameter value over others. Adaptive behavior in an environment that emphasizes social relations and responsibility is likely to require a rather global attentional focus in many situations, whereas an environment emphasizing the individual and his/her separation from others arguably calls for behavior that is based on a rather local focus. It makes sense to assume that individuals raised in these environments acquire chronic biases towards particular parameters (Hommel and Colzato, 2010), if we assume that behavior that heeds the expectations of the social environment is selectively rewarded, and if we assume that this favors and promotes learning of the control parameters that generate this behavior (Waszak et al., 2003). This does not preclude the selection of other parameters if the current task requires so. However, processing local and global aspects of stimulus events using other than the preferred parameter values comes with a cost in time and effort.

The present study aimed to overcome two major limitations of previous research on the impact of religious practice on attentional control (Colzato et al., 2008, in press). For one, the previous studies were all restricted to European- or Europe-oriented countries (e.g., Israel). Despite some variation, many of these countries have a relatively strong emphasis on individualism, as indicated by rather high scores on Hofstede's (2001) National Individualism scale (i.e., 80, 76, and 54 from 100 for The Netherlands, Italy, and Israel, respectively). To demonstrate a specific impact of religious practice that goes beyond general national or cultural habits and attitudes, it is important to show that religious practice impacts the global-precedence effect in a way that is independent of the cultural peculiarities of a given country or group. In particular it would seem important to show that the effect of religious practice on attentional bias is similar in countries scoring high and countries scoring low on individualism.

Although Japanese participants are frequently included in intercultural comparisons with US Americans (see Nisbett and Miyamoto, 2005), Japan is one of the most individual-oriented countries in Asia (Hofstede's Individualism score =46). Therefore, we opted for recruiting Taiwanese participants, who are assumed to have a decidedly collectivistic orientation (Individualism score $=17$ ).

The second limitation our study aims to overcome relates to the type of religion. All religions considered thus far have a more or less European origin and a strongly "Western" flavor. They are associated with official, published doctrines (sacred writings and "rule books") that prescribe and, to quite some degree, describe daily religious practice. This is not typical for many other religions, especially those being followed in the Eastern hemisphere. A prominent example is Buddhism, where the link between doctrine and practice is often less strict and pronounced for lay practitioners, and which emphasizes values that are very different from the more Western religions. Nevertheless, Buddhism does emphasize the physical and social context the practitioner lives in (Guenther, 1993), which, according to our hypothesis, should propagate a global attentional bias.

Despite the popular impression, most traditional Buddhists (in Asia) do not practice formal meditation techniques (e.g., breath awareness meditation); neither is there necessarily anything particularly Buddhist about meditation, but it is true that Buddhist authors, philosophers, and mystics have written much about meditation and theorized much about the functioning of the mind (Colzato and Silk, 2010). The religious practice of most traditional Buddhists in Asia consists, hence, not in meditating but in daily praying, weekly visiting the temple, writing, reading, and reciting Sutra calligraphy and living for short period in a monastery.

To summarize, we compared Taiwanese Zen Buddhists with Taiwanese atheists with regard to their performance on Navon's (1977) global-local task. If Zen Buddhist practice results in a chronic bias towards a more global control parameter, then Zen Buddhists would show a greater global-precedence effect.

\section{MATERIALS AND METHODS PARTICIPANTS}

We tested 41 young healthy adults, who participated for partial fulfillment of course credit or a financial reward (NT\$250= US\$8). They constituted two experimental groups: Atheists and Zen Buddhists (all active members of Buddhist organizations such as the Tzu Chi Foundation, Bliss Wisdom Club, and Amida Society). All participants were matched in terms of ethnicity (100\% the yellow race), Culture (100\% Taiwanese), age, sex, and IQ (measured by Raven's standard progressive matrices: SPM, Raven et al., 1988) - see Table 1 for demographic data and religious behavior. Both groups were educated in Taiwan according to the same educational style, and reported similar social-economical background. Written informed consent was obtained from all participants after the nature of the study was explained to them.

\section{APPARATUS AND STIMULI}

Responses were made by pressing the "Z" or "?" of the QWERTY computer keyboard with the left and right index finger, respectively. The target stimuli were adopted from Huizinga et al. (2006), and consisted of geometric figures (see Figure 1). Larger (global) rectangles or squares consisted of smaller (local) rectangles or squares. Global stimuli (i.e., squares or rectangles; $93 \times 93$ pixels or $93 \times 189$ pixels respectively) were composed of many smaller "local" stimuli (i.e., squares or rectangles; $21 \times 21$ pixels or $8 \times 46$ pixels respectively). The space between the local elements of a stimulus was three pixels. A global square consisted of 16 small squares or eight small rectangles; a global rectangle consisted of 32 small squares or 16 small rectangles.

\section{TASK AND PROCEDURE}

Participants responded to randomly presented rectangles or squares by pressing the left or right response key, respectively. Three blocks of trials were administered, two training blocks in which the instruction (global or local) was constant across all trials, followed by the experimental block in which participants switched between the global and the local task. In one of the two training blocks, participants responded to the local figure, in the other block they responded to the global figure. The order of the training blocks was randomized across participants and each block consisted of 50 trials. In the third block, consisting of 160 trials, participants alternated between predictable sequences of four "local" and four "global" trials). A cue indicated to which dimension (global or local) the participants should respond. Cues that related to the global 
Table 1 | Demographic characteristics and religious behavior of participants, and performance on globally and locally defined targets.

\begin{tabular}{|c|c|c|}
\hline Variables (SD) & Buddhists & Atheists \\
\hline Sample $N(\mathrm{M}: \mathrm{F})^{*}$ & $20(9: 11)$ & $21(13: 8)$ \\
\hline Age (years) & $23.2(4.8)$ & $21.4(2.0)$ \\
\hline Raven IQ & $115.7(3.3)$ & $116.9(2.3$ \\
\hline \multicolumn{2}{|l|}{ groups $(Y e s: N o)^{* *}$} & $0: 21$ \\
\hline \multicolumn{3}{|l|}{ vegetarian $\left(\right.$ Yes:No) ${ }^{* *}$} \\
\hline \multicolumn{2}{|l|}{ religious activities (Yes:No) ${ }^{* *}$} & $0: 21$ \\
\hline Visit to temple (weekly) (Yes:No) ${ }^{* *}$ & 20:0 & $0: 21$ \\
\hline Zen meditation (Yes:No) & $0: 20$ & $0: 21$ \\
\hline Prayer (daily) $($ Yes:No)** & $18: 2$ & $0: 21$ \\
\hline Sutra calligraphy (Yes:No)* & $5: 15$ & $0: 21$ \\
\hline Sutra reading* & $5: 15$ & $0: 21$ \\
\hline Sutra recitation* & $5: 15$ & $0: 21$ \\
\hline Short-term living in a monastery (Yes:No) ${ }^{*}$ & $4: 16$ & $0: 21$ \\
\hline $\begin{array}{l}\text { Members of Buddhism activities } \\
\text { staff/administrative position in temple* }\end{array}$ & $4: 16$ & $0: 21$ \\
\hline Volunteers in social services* & 4:16 & $0: 21$ \\
\hline \multicolumn{3}{|l|}{ SOCIAL VALUE ORIENTATION CATEGORY } \\
\hline Prosocial* & 13 & 7 \\
\hline Individualistic & 6 & 7 \\
\hline Competitive & 0 & 5 \\
\hline Unclassified & 1 & 2 \\
\hline \multicolumn{3}{|l|}{ GLOBAL TARGETS } \\
\hline Reaction times (ms) & $358(9.7)$ & $351(9.5)$ \\
\hline Error rates $(\%)$ & $1.9(0.8)$ & $4.2(0.8)$ \\
\hline \multicolumn{3}{|l|}{ LOCAL TARGETS } \\
\hline Reaction times (ms) & $436(11.1)$ & 404 (10.9) \\
\hline Error rates (\%) & $7.6(1.5)$ & $5.8(1.4)$ \\
\hline \multicolumn{3}{|l|}{ GLOBAL PRECEDENCE } \\
\hline Reaction times $(\mathrm{ms})^{* *}$ & 78 & 53 \\
\hline Error rates (\%) & 5.7 & 1.6 \\
\hline
\end{tabular}

Standard errors are presented in parentheses. Significant group difference; ${ }^{*} p<0.05,{ }^{*} p<0.01$.

(local) dimension consisted of a big (small) square, presented at one side of the target stimulus, and a big (small) rectangle, presented at the other side of the target stimulus. The subjects were instructed to react as fast as possible. The color of cues and target was red. Both remained on the screen until a response was given or $2500 \mathrm{~ms}$ had passed. The time interval between presentation of the cue and of the target stimulus varied between 400 and $500 \mathrm{~ms}$ and the interval between responses and the next presentation of the cue varied between 900 and $1100 \mathrm{~ms}$.

All participants were tested individually and completed the intelligence test, the global-local task, and the social value orientation task. Individual IQ was determined by means of a 30-min reasoning-based intelligence test (Raven's SPM; Raven et al., 1988). The SPM assesses the individual's ability to create perceptual relations and to reason by analogy independent of language and formal schooling; it is a standard, widely used test to measure Spearman's g factor and of fluid intelligence in particular. A Chinese translation of the social value orientation task (van Lange et al., 1997) was used in order quantify prosocial, individualistic, and competitive behaviors. This task consists of series of decomposed games, involving choices among combinations of outcomes for oneself, and for another person to whom hypothetical points are assigned.

\section{STATISTICAL ANALYSIS}

$t$-Tests and Chi-square tests were performed to test for group effects in Age, IQ, and social value orientation scores. Following Huizinga et al. (2006) and Colzato et al. (2008), median reaction time (RT) and square root error percentages were analyzed by means of ANOVAs using target level (global vs. local), Congruency between the stimuli on the two levels (congruent vs. incongruent), and Task Switch (i.e., same vs. different target level with respect to the previous trial; task repetition vs. alternation) as within- and group (Zen Buddhists vs. Atheists) as between-participants factor. A significance level of $p<0.05$ was adopted for all tests.

\section{RESULTS}

Analysis of social value orientation revealed significant differences between Zen Buddhists and Atheists participants on the variable prosocial behavior, $\chi^{2}=7.35, \mathrm{df}=3, p=0.04$; Zen Buddhists were oriented to prosocial behavior to a larger extend than Atheists. No significant group differences were obtained for age, $t(39)=1.57$, $p>0.05$, intelligence, $t(39)=-1.36, p>0.05$, and sex, $t(39)=1.07$, $p>0.05$.

Reaction time analysis showed a main effect of Target Level, $F(1,39)=247.80, p<0.0001$, MSE $=350.127, \eta_{p}^{2}=0.86$, which was modified by group, $F(2,39)=9.29, p<0.05$, MSE $=350.127$, $\eta_{p}^{2}=0.19$. As confirmed by follow-up ANOVAs, both Zen Buddhists and Atheists responded significantly faster to global than to local targets, $F(1,19)=125.25, p<0.0001, \mathrm{MSE}=481.701, \eta_{p}^{2}=0.87$; and $F(1,20)=128.43, p<0.0001, \mathrm{MSE}=225.131, \eta_{p}^{2}=0.86$, respectively. However, this global-precedence effect was notably larger for Buddhists than it was for Atheists (see Figure 2). Error percentages revealed a reliable main effect of target level only, $F(1,59)=19.63$, $p<0.0001, \mathrm{MSE}=25.90, \eta_{p}^{2}=.25$, indicating fewer errors on global than on local target trials.

A second, more explorative ANOVA on RT tested for group effects of task switch - comparing trials where the current target level matched the target level on the previous trial with trials with a switch between levels - and Congruency between levels-comparing trials on which the global and the local target matched with trials on which they did not. Four factors were considered: group (Zen Buddhists vs. Atheists), target level (global vs. local), task switch (repetition vs. switch of target level), and Congruence (same global and local target vs. different). The only reliable effect involving the group factor was a three-way interaction between group, target level, and congruency, $F(1,39)=5.40, p<0.03, \mathrm{MSE}=2544.38$, $\eta_{p}^{2}=0.12$. The underlying pattern showed that Congruency had no impact on responding to global targets ( -5 and $5 \mathrm{~ms}$ congruency effects in Zen Buddhists and Atheists, respectively) but had a stronger effect on Zen Buddhists (41 ms) than on Atheists $(29 \mathrm{~ms})$ when responding to local targets. 


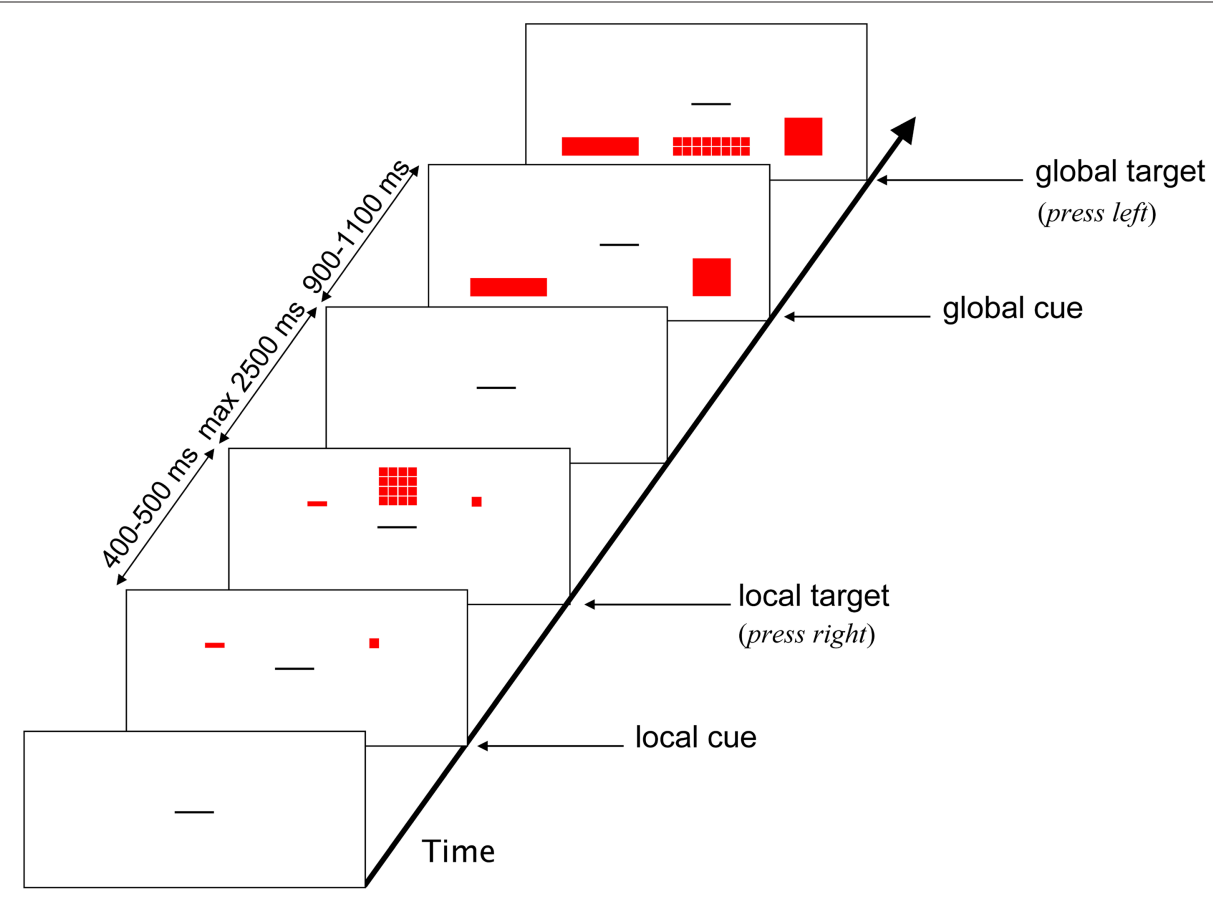

FIGURE 1 | Sequence of events for a local and a global target trial.

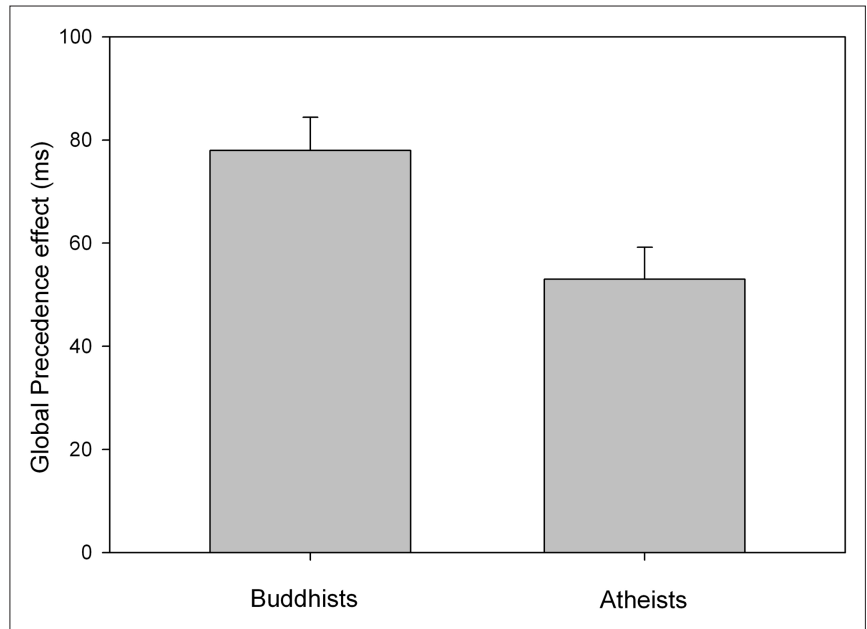

FIGURE 2 | Mean global-precedence effect for Atheists and Buddhists. Vertical capped lines atop bars indicate standard error of the mean.

\section{DISCUSSION}

Our findings show that Zen Buddhists and Atheists differ in the way they attend to global and local features of visual stimuli. As expected, Buddhists were associated with a more pronounced global-precedence effect compared to Atheists, indicating that followers of this believe system - which is based on the concept of compassion and open presence (Guenther, 1993) - seem to have a systematic bias towards more global aspects of perceived events. Consistent with our hypothesis that this bias might be a reflection of the Buddhist emphasis on "compassion" with the physical and social context (even if in this study we did not directly measure compassion), Buddhists were significantly more oriented towards prosocial behavior than Atheists.

Given that our subjects, as the most traditional Buddhists in Asia, did not meditate and that there is nothing particular Buddhist about meditation, we assume that the effect observed may be due to religious practice as prayers, temple visit and being active member of Buddhist organizations.

We recognize the alternative possibility that it is not religion, per se, which is responsible for the obtained difference but some other social, motivational, or personality characteristic that is correlated with religion. For example, one could argue that Buddhism is more attractive for people with a global attentional bias, so that the findings may reflect mere self-selection. However, people commonly join religious groups long before attentional or other biases become obvious (often by birth, following family traditions), which makes self-selection relatively unlikely. Moreover, we controlled for, and thus ruled out, group differences with respect to intelligence, age, mood, several personality traits, educational style, cultural background, and socio-economic status. This does not seem to leave obvious alternative candidates to explain our findings, even though the correlative nature of our study is unable to rule out possible alternatives in principle.

In addition to showing a more pronounced precedence effect, Zen Buddhists were also more affected by congruency than Atheists. This effect was restricted to local targets, which is consistent with the standard finding that global processing is more immune to local interference than local processing is to global interference (Navon, 1977). When processing local targets, Zen Buddhists thus seem to have greater difficulty to ignore global information, even if this information is misleading and suggesting the wrong response. 
Like the larger global-precedence effect, this greater sensitivity to global information suggests that Zen Buddhists have a chronic bias towards global information. Nevertheless, Zen Buddhists were quite capable of responding to local targets, which shows that their global preference can be overcome. However, this requires extra efforts and is more difficult to achieve, as suggested by the impact of congruency on performance.

Even though most traditional Buddhists (in Asia) do not meditate, it would be very interesting to investigate whether practitioners of Vipassana meditation (based on open monitoring) would be associated with a more pronounced global-precedence effect than practitioners of Samatha (based on focused attention).

The present findings support the idea that one's religious practice can create systematic and stable preferences for a particular range of parameter values used to configure one's cognitive system (Colzato et al., in press; Hommel and Colzato, 2010). In other words, religion can create specific cognitive-control profiles that are

\section{REFERENCES}

Boesak, A. (1984). Apartheid, Liberation and the Calvinist Tradition. Johannesburg: Skotaville Publications.

Bratt, J. D. (1998). Abraham Kuyper: A Centennial Reader. Grand Rapids, MI: Eerdmans.

Colzato, L. S., and Silk, J. (2010). Editorial: imag(in)ing the Buddhist brain.Zygon: J. Relig. Sci. 45, 591-595.

Colzato, L. S., van Beest, I., van den Wildenberg, W. P. M., Scorolli, C., Borghi, A. M., Dorchin, S., Meiran, N., and Hommel, B. (2010). God: do I have your attention? Cognition, 117, 87-94.

Colzato, L. S., van den Wildenberg, W. P. M., and Hommel, B. (2008). Losing the big picture: how religion may control visual attention? PLoS One 3: e3679. doi:10.1371/journal. pone. 0003679 .

Guenther, H. V. (1993). Ecstatic Spontaneity: Saraha's Three Cycles of Doha. Freemont, CA: Asian Humanities Press.
Hofstede,G.(2001).Culture's Consequences: Comparing Values, Behaviors, Institutions, and Organisations Across Nations. Thousand Oaks, CA: Sage.

Hommel, B., and Colzato, L. S. (2010). Religion as a control guide: on the impact of religion on cognition. Zygon: J. Relig. Sci. 45, 596-604.

Huizinga, M., Dolan, C. V., and van der Molen, M. W. (2006). Age-related change in executive function: developmental trends and a latent variables analysis. Neuropsychologia 44, 2017-2036.

Logan, G. D. (1996). The CODE theory of visual attention: an integration of space-based and object-based attention. Psychol. Rev. 103, 603-649.

Logan, G. D., and Gordon, R. D. (2001). Executive control of visual attention in dual-task situations. Psychol. Rev. 108, 393-434.

Masuda, T., and Nisbett, R. E. (2001). Attending holistically versus analytically: comparing the context sensitivity of Japanese and Americans. J. Pers. Soc. Psychol. 81, 922-934. shared by its members. These profiles shape information processing even in tasks that are unrelated to, and irrelevant for religious values and practice. The fact that the impact of such profiles can be observed both in individualistic cultures (Colzato et al., 2008, in press) and, as in the present study, in collectivistic cultures suggests that it is not just cultural orientation that matters but point to the contribution of specific values and practice. This concept may call for a reinterpretation of previous comparisons between US-Americans and Japanese participants (Nisbett and Miyamoto, 2005), where religion was not controlled for.

\section{ACKNOWLEDGMENTS}

We thank Ya-Ting Huang for her invaluable assistance in recruiting, testing the participants of this study, and collecting the data. The research of Lorenza S. Colzato and Wery P. M. van den Wildenberg is supported by NWO (Netherlands Organization for Scientific Research).

McKone, E., Davies, A. A., Fernando, D., Aalders, R., Leung, H. Wickramariyaratne, T., and Platow, M J. (2010). Asia has the global advantage: race and visual attention. Vision Res. 50, 1540-1549.

Navon, D. (1977). Forest before trees: the precedence of global features in visual perception. Cogn. Psychol. 9, 353-383.

Nisbett, R. E., and Miyamoto, Y. (2005). The influence of culture: holistic versus analytic perception. Trends. Cogn. Sci. 9, 467-473.

Raven,J.C., Court,J.H., and Raven,J. (1988). Manual for Raven's Progressive Matrices and Vocabulary Scales. London: Lewis.

van Lange, P. A. M., Otten, W., de Bruin, E., and Joireman, J. A. (1997). Development of prosocial, individualistic, and competitive orientations: theory and preliminary evidence. $J$. Pers. Soc. Psychol. 73, 733-746.

Waszak, F., Hommel, B., and Allport, A. (2003). Task-switching and long-term priming: role of episodic stimulus-task bindings in task-shift costs. Cogn Psychol. 46, 361-413.
Conflict of Interest Statement: The authors declare that the research was conducted in the absence of any commercial or financial relationships that could be construed as a potential conflict of interest.

Received: 21 May 2010; paper pending published: 28 June 2010; accepted: 02 September 2010; published online: 20 September 2010.

Citation: Colzato LS, Hommel B, van den Wildenberg WPM and Hsieh S (2010) Buddha as an eye opener: a link between prosocial attitude and attentional control. Front. Psychology 1:156. doi:10.3389/ fpsyg.2010.00156

This article was submitted to Frontiers in Cognition, a specialty of Frontiers in Psychology.

Copyright (c) 2010 Colzato, Hommel, van den Wildenberg and Hsieh. This is an open-access article subject to an exclusive license agreement between the authors and the Frontiers Research Foundation, which reproduction in any medium, provided the original authors and source are credited. permits unrestricted use, distribution, and 